\title{
Psychosocial, Economic Support Services and Demographic Factors Affecting the Prognosis of Schizophrenic Clients at Adama Hospital Medical College
}

\author{
Nuru Hassen Ibrahim ${ }^{1}$, Kumsa Donis ${ }^{2}$ \\ ${ }^{1}$ School of Social and Public Department, Adama Hospital Medical College, Adama City, Ethiopia \\ ${ }^{2}$ School of Psychology, Adama Science and Technology University, Adama City, Ethiopia
}

Email address:

nurulove27@gmail.com (N. H. Ibrahim)

\section{To cite this article:}

Nuru Hassen Ibrahim, Kumsa Donis. Psychosocial, Economic Support Services and Demographic Factors Affecting the Prognosis of Schizophrenic Clients at Adama Hospital Medical College. American Journal of Psychiatry and Neuroscience. Vol. 4, No. 4, 2016 , pp. 57-64. doi: 10.11648/j.ajpn.20160404.11

Received: May 5, 2016; Accepted: May 16, 2016; Published: June 18, 2016

\begin{abstract}
The aim of this study is to identify the psychosocial, economic support services and demographic factors affecting the prognosis of schizophrenic clients at Adama Hospital medical college, Oromia region, Ethiopia. To conduct the study, quantitative method was employed and a survey was conducted from February to march 2014. A sample of 100 schizophrenic clients who are on follow-up with antipsychotic medication for greater than one year was selected by using psychiatric nurse and mental health officer for their cooperative communication using mental state examination to assess their cognition before delivering questionnaire for the study'. For the study of the psychosocial, economic support service and demographic factors affecting the prognosis of schizophrenia, questionnaire was developed according to the objective of the study and translated into local language (Amharic language). To ensure the validity and reliability of developed questionnaire various measures were taken and pilot study was conducted at the study site. In accordance with finding of pretest, minor modifications were made to the questionnaire. The data were analyzed using frequency, percentage, through SPSS version 16.0 statistical packages. The results showed that $30 \%$ clients were below age 25 years, $18 \%$ of schizophrenic clients are illiterate, $38 \%$ of them are grade $1-8,31 \%$ of them are grade $8-12$, and $3 \%$ of them are certificate. The $74 \%$ of schizophrenic client's monthly income is below $\$ 27,49 \%$ of schizophrenic client are unmarried, $13 \%$ of them are separated and divorced, and $1 \%$ of them are widowed. With regard to occupational status $52 \%$ of schizophrenic's client are unemployed, $1 \%$ dismissed, $10 \%$ of them are house holder and $20 \%$ of them are daily laborer. $43 \%$ of clients discontinued taking the prescribed medication, $16 \%$ of the respondents reported that they had experienced stigma in their life due to schizophrenia disorder. The findings of the study showed that, the psychosocial, economic support services and demographic factors affecting the prognosis of schizophrenia disorder is assessed and identified in the study area. This needs an urgent attention at all levels of societal hierarchy including minsters, administrators, stakeholders, health professionals, and the community to overcome the problem. Finally, recommendations were forwarded based on the major findings so as to minimize the problem.
\end{abstract}

Keywords: Prognosis of Schizophrenia, Psychosocial, Economic Support Service, Demographic Factors

\section{Introduction}

"Schizophrenia is the label given to a group of psychoses in which deterioration of functioning is marked by severe distortion of thought, perception, mood, bizarre behavior, and social withdrawal [1]". Schizophrenia can affect anybody, with no class or strata of people being exempt. "Doctors, chartered accountants, lawyers, educated and uneducated people, men and women, rich and poor, urban and rural, young and old, can all be afflicted with schizophrenia [3]". Both patients and their families often suffer from poor care and social ostracism because of widespread ignorance about the disorder. "Although schizophrenia is discussed as if it is a single disease, it probably comprises a group of disorders with heterogeneous etiologies, and it includes patients whose clinical presentations, treatment response, and courses of 
illness vary [2]".

"Epidemiologically, the lifetime prevalence of schizophrenia is about one percent. About 0.05 percent of the total population is treated for schizophrenia in any single year, and only about half of all patients with schizophrenia obtain treatment, despite the severity of the disorder. In Ethiopia, the prevalence of schizophrenia is $0.7 \%$ [4]".

Schizophrenia is equally prevalent in men and women. The two genders differ, however, in the onset and course of illness. Onset is earlier in men than in women. More than half of all male schizophrenia patients, but only one-third of all female schizophrenia patients, are first admitted to a psychiatric hospital before age 25. "The peak ages of onset are 10 to 25 years for men and 25 to 35 years for women because schizophrenia appear early on male than women. Onset of schizophrenia before age 10 or after age 60 is extremely rare, due to pathophysiologic nature of the disorder [2]".

In 2013, the American Psychiatric Association released the fifth edition of the Diagnostic and statistical manual [7]" useful in the diagnosis of schizophrenia. "To be diagnosed with schizophrenia, two diagnostic criteria have to be met over much of the time of a period of at least one month, with a significant impact on social or occupational functioning for at least six months. The person had to be suffering from delusions, hallucinations or disorganized speech. A second symptom could be negative symptoms or severely disorganized or catatonic behavior. The definition of schizophrenia remained essentially the same as that specified by the 2000 version of Diagnostic and Statistical Manual IVTR, but Diagnostic and Statistical Manual-V makes a number of changes [7]".

Antipsychotic medications are effective in treating psychotic symptoms and hallucinations. However, some of the patients experience adverse side effects such as drowsiness, blurred vision, skin rushes or menstrual issues for women. Psychosocial treatments and social support are of great assistance to patients who have been successful in using antipsychotic medication. They assist patients to exhibit normal day-to-day behaviors, (NHIM, 2010). "Some evidences indicate that regular exercise has a positive effect on the physical and mental health of those with schizophrenia, [8]".

"The first-line psychiatric treatment for schizophrenia is antipsychotic medication, which can reduce the positive symptoms of psychosis in about 7-14 days. There are two types of antipsychotic; the typical antipsychotics are chlorpromazine, haloperidol, trifluperazin and fluphenazin which are also called first generation antipsychotic drugs. Clozapin, risperidone and olanzapins are atypical antipsychotic or second generation antipsychotic drugs for schizophrenia [2]".

"For people who are unwilling or unable to take medication regularly, long-acting depot preparations of antipsychotics may be used to achieve control. They reduce the risk of relapse to a greater degree than oral medications. When used in combination with psychosocial interventions they may improve long-term adherence to treatment. The American Psychiatric Association suggests considering stopping antipsychotics in some people if there are no symptoms for more than a year [12]".

"A number of psychosocial interventions may be useful in the treatment of schizophrenia including: family therapy, assertive community treatment, supported employment, cognitive remediation, skills training, cognitive behavioral therapy, token economic interventions, and psychosocial interventions for substance use and weight management. Family therapy or education, which addresses the whole family system of an individual, may reduce relapses and hospitalizations [8]".

Evidence shows that adding psychosocial treatments to medication therapy results in better outcomes than either medication alone or psychosocial therapy alone. For example, people who are treated with a combination of medication, social skills training, and family therapy have only a five percent chance of relapse, compared to a forty percent chance of relapse among those treated with medications alone. "Taking advantage of various psychosocial approaches is not a matter of choosing one over another, or using them as substitutes for medication. For the practical and optimal treatment of schizophrenia, it's advantageous to combine medication and psychosocial approaches that enhance once another [8]".

"The prognosis for patients diagnosed with schizophrenia varies [9]". Factors which are responsible for a good prognostic outcome of schizophrenia are primarily the older the patient, the shorter the duration prior to treatment, the more speedily the symptoms develop, and patient who had close friendships and multiple relationships prior to illness [3]". An episode brought on by a major identifiable life stress will respond more quickly than an episode without any obvious cause [9]". A patient with a stable and helpful marital partner has a favorable prognosis as compared to an unmarried patient [2]". The higher the level of education, the more is the chance of a patient coming rapidly to terms with the illness and handling the post - illness sequence [3]". "Patients with a good stable occupation or business prior to onset of illness will respond better than a patient who is jobless and economically unsound and absence of schizophrenia in the family points to a better prognosis [2]". "Finally hostile behavior by family members or vice versa, excessive care and attention by them can undermine the patient's sense of confidence and hamper recovery and patient with a joint family and a staunch circle of friends who are ready lend a helping hand, is much better off than alone man afflicted with the illness, whose relatives are in some far off land, and who has no one to turn too [3]".

The effects of schizophrenia on a person's life experience and opportunities are considerable; service users and careers need help and support to deal with their future and to cope with any changes that may happen regarding psychosocial, economic supply services and demographic factors affecting prognosis of schizophrenia. 


\section{Research Question}

The main research question is "what are the psychosocial, economic support services and demographic factors that affect the prognosis of schizophrenia at Adama Hospital Medical College?

\subsection{Sub Research Questions Are}

1. What are the psychological supports services affecting the prognosis of schizophrenia?

2. What are the social supports services affecting the prognosis of schizophrenia?

3. What are the economic supports services affecting the prognosis of schizophrenia?

4. What are the demographic factors affecting the prognosis of schizophrenia?

\subsection{Objective of the Study}

The major objective of the study is to identify psychosocial, economic support services and demographic factors affecting the prognosis of schizophrenic clients. The specific objectives of the study are as follows:

- To assess the psychological support services in the prognosis of schizophrenia.

- To assess the social support services that affects the prognosis schizophrenic clients.

- To identify the economic support services that affects the prognosis of schizophrenic clients.

- To determine the demographic factors in the prognosis of schizophrenic client.

\section{Methodology}

\subsection{Research Design}

This research paper is based on cross sectional study designs to address the objectives of the study: to assess and identify the psychosocial, economic supply services and demographic factors affecting the prognosis of schizophrenia disorder. A survey study portrays the status of a phenomenon at a particular time, determines what happened during a given activity and deals with the relationships between variables. It mainly focuses on research design, test and measurement procedure of a reality and statistical analysis to respond to research questions requiring numerical data (Williams, 2007:65). Through the application of a survey, generalizations can be developed (Best \& Kahn, 2006:118; Leedy \& Ormrod, 2001:102). The selection of the use of a survey is, therefore, to generalize the findings from the sample to the entire population in the selected area. In addition systematic review and descriptive analysis was employed to see the psychosocial, economic supply services and demographic factors affecting the prognosis of schizophrenia.

\subsection{Population}

All schizophrenic clients on medication at Adama Hospital medical college from Adama city and outreach are the target of the study. "From the total population, $1 \%$ of them are schizophrenic worldwide and about 0.05 of the total population is treated for schizophrenia in any singly year as indicated in [2]". "In Ethiopia, $0.7 \%$ of the total populations are schizophrenics [5]". It is estimated that 339 of Adama city population and outreach areas are victims of schizophrenic. From the total victims of schizophrenic clients, $0.05 \%$ (242) treated in a single year in which only half of them are on treatment for greater than one year as the six months report of Adama Hospital medical college psychiatric clinic 2013 report reveals.

\subsection{The Sample}

The source population to address inclusion criteria to the objectives of study was all communicative and ethically cooperative schizophrenic clients' on medication for greater than one year in the study area. The selection of these clients was made from diagnosis written on patient card in psychiatric clinic. Thus, the total number of clients that the researcher enrolled in this study was one hundred fifty samples with the expectation that some of them are non-responsive. Thus, 100 schizophrenic clients were found to be responsive and they were interviewed by self - administered questionnaire for forty five days. The reason for choosing this particular group of population is that prognosis of schizophrenia is not identified till one year of the therapy. Schizophrenic client in loosening of association, non -cooperative due to suspicious nature of the disorder and clients bellow one year of therapy are excluded from in the study.

\subsection{Instrumentation}

For the study of the psychosocial, economic factors affecting the prognosis of schizophrenia, questionnaire was developed from literature review according to the objective of the study. The questionnaire was used because these types of question format provide the frame of reference for all respondents to use in determining their answers. The questionnaire was used as a means to measure whether any treatments for schizophrenia were sought. The questionnaire ask respondent to indicate for each of the behavior that are manifested. The instrument was also designed to determine psychosocial, economic supply services and demographic factors affecting prognosis of schizophrenia. It is further used to determine how parents perceive emotional problem of schizophrenic clients, and to manage chronic problem of the schizophrenic client. The developed questionnaire has three parts and the strength of the survey questionnaire was comprehensive; all measuring scales are applied yet manageable for clients to read and complete. Validity of the developed questionnaire was tested by five experts who have second degree and higher (phd in psychology, Msc in mental health officer, and three of them with $\mathrm{Mph}$ ). The translation of the questionnaire from English to Amharic was done and then converted to English language by mental health professional to check for language equivalence. Pilot study was also conducted prior to the main data collection for $10 \%$ 
of schizophrenic clients and analyzed to check the reliability of the instruments. The translated questionnaire was tested for feasibility of use, acceptability, and reliability. The assessment showed that the questionnaire was acceptable and feasible with high reliability of $\alpha 0.87$. In accordance with finding of pretest, minor modifications were made to the questionnaire. The question and statements were grouped and arranged according to proposed objective. The response was quite good, given the nature of the questions being asked and the effort required completing the survey.

\subsection{Data Processing and Analysis}

With regard to data management, all completed questionnaire forms are reviewed by data collector and checked by researcher. Forms with missing data were returned to the interviewer immediately for correction. The data were categorized and coded on well-defined coding sheet. Later it was exported to SPSS statistical software for window version 16 for analysis. For the analysis of the psychosocial, economic supply services and demographic factors affecting the prognosis of schizophrenia descriptive is the unit of analysis. The analysis focused on the pretest conceptual analysis. Thus descriptive analyses were done based on the objective of the study. Finally the results of the analyses were presented using frequency and percentage.

\subsection{Ethical Consideration}

To conduct the study, the research proposal was examined and screened for scientific and ethical clearance by institutions research highest body for approving research in the college of medical science, Adama Hospital Medical College. Prior to administering the survey and collecting data, permission letter was obtained from Adama science and Technology University, Adama Hospital medical college and authorities of different levels including the area where the study was conduct. The researcher also asked permission from respondents' parent and schizophrenic client to gain full commitment about the study. (In our culture majority of schizophrenic client appear to psychiatric clinic with their relatives. So in order to collect the data both of them must give their permission, otherwise chaos may appear and considered as an abuse. After getting their permission only clients are allowed to fill the questionnaires', but not the relatives. Finally the outcome result presented to all schizophrenic parents at nongovernmental organization who support them in the form of discussion. The selected schizophrenic clients were given clear information about the study and each voluntary participant signed out on consent prepared in local language. Confidentiality is intact and clients were informed that they have full right to refuse to participate in the study at any time during assessment.

\section{Result and Discussion}

\subsection{Results}

Table 1. The demographic information of the respondents.

\begin{tabular}{|c|c|c|c|}
\hline No. & Demographic variable & Frequency & Percentage \\
\hline \multirow[t]{4}{*}{1} & Sex & & \\
\hline & Male & 51 & 51 \\
\hline & Female & 49 & 49 \\
\hline & Total & 100 & 100 \\
\hline \multirow[t]{5}{*}{2} & Age & & \\
\hline & $10-25$ & 30 & 30 \\
\hline & $26-45$ & 58 & 58 \\
\hline & Above 45 & 12 & 12 \\
\hline & Total & 100 & 100 \\
\hline \multirow[t]{10}{*}{3} & Education & & \\
\hline & Illiterate & 18 & 18 \\
\hline & $1-8$ grade & 38 & 38 \\
\hline & $9-12$ & 31 & 31 \\
\hline & Certificate & 3 & 3 \\
\hline & Diploma & 9 & 9 \\
\hline & Bachelor degree & 0 & 0 \\
\hline & Master degree & 0 & 0 \\
\hline & $\mathrm{PhD}$ & 1 & 1 \\
\hline & Total & 100 & 100 \\
\hline \multirow[t]{6}{*}{4} & Monthly income & & \\
\hline & $<500$ birr & 74 & 74 \\
\hline & $501-1000$ & 16 & 16 \\
\hline & $1001-2000$ & 5 & 5 \\
\hline & $>2000$ & 5 & 5 \\
\hline & Total & 100 & 100 \\
\hline \multirow[t]{7}{*}{5} & Marital status & & \\
\hline & Single & 49 & 49 \\
\hline & Married & 34 & 34 \\
\hline & Separated/divorced & 13 & 13 \\
\hline & Widowed & 3 & 3 \\
\hline & If others (specify) & 1 & 1 \\
\hline & Total & 100 & 100 \\
\hline \multirow[t]{7}{*}{6} & Occupation & & \\
\hline & Unemployed & 52 & 52 \\
\hline & Employed & 17 & 17 \\
\hline & Dismissed & 1 & 1 \\
\hline & House wife & 10 & 10 \\
\hline & If others & 20 & 20 \\
\hline & Total & 100 & 100 \\
\hline
\end{tabular}

Table 2. Frequency of reasons for discontinuation of medication.

\begin{tabular}{llll}
\hline Item & Frequency & Percent & \\
\hline Needs of traditional therapy & 15 & 35 \\
Lack of money & 10 & 23 \\
Drugs not available & 3 & 7 \\
Mention (if others) & 15 & 35 \\
Total & 43 & 100.0 \\
\hline
\end{tabular}


Table 3. Types of stigma that schizophrenic client experienced.

\begin{tabular}{lll}
\hline Experienced stigma & Frequency & Percentage \\
\hline Social isolation & 7 & 43.75 \\
Alienation & 4 & 25 \\
Perceived discrimination & 3 & 18.75 \\
Stereotyped endorsement & 2 & 12.5 \\
Total & 16 & 100 \\
\hline
\end{tabular}

Table 4. Frequency of consequences of stigma that schizophrenic client manifest.

\begin{tabular}{lll}
\hline Consequences of stigma & Frequency & Percentage \\
\hline Social isolation & 5 & 31.25 \\
Alienation & 4 & 25 \\
Discrimination & 1 & 6.25 \\
Stereotyped endorsement & 6 & 37.5 \\
Total & 16 & 100 \\
\hline
\end{tabular}

Table 5. Premorbid good functioning.

\begin{tabular}{llll}
\hline Item & Frequency & Percentage \\
\hline Very good functioning & $\overline{79}$ & $\overline{79}$ & \\
Good functioning & 21 & 21 & \\
Not functioning & 21 & 2 \\
\hline
\end{tabular}

Table 6. Current functioning.

\begin{tabular}{lll}
\hline Item & Frequency & Percentage \\
\hline Very good functioning & 15 & 15 \\
Good functioning & 45 & 45 \\
Not functioning & 40 & 40 \\
Total & 100 & 100 \\
\hline
\end{tabular}

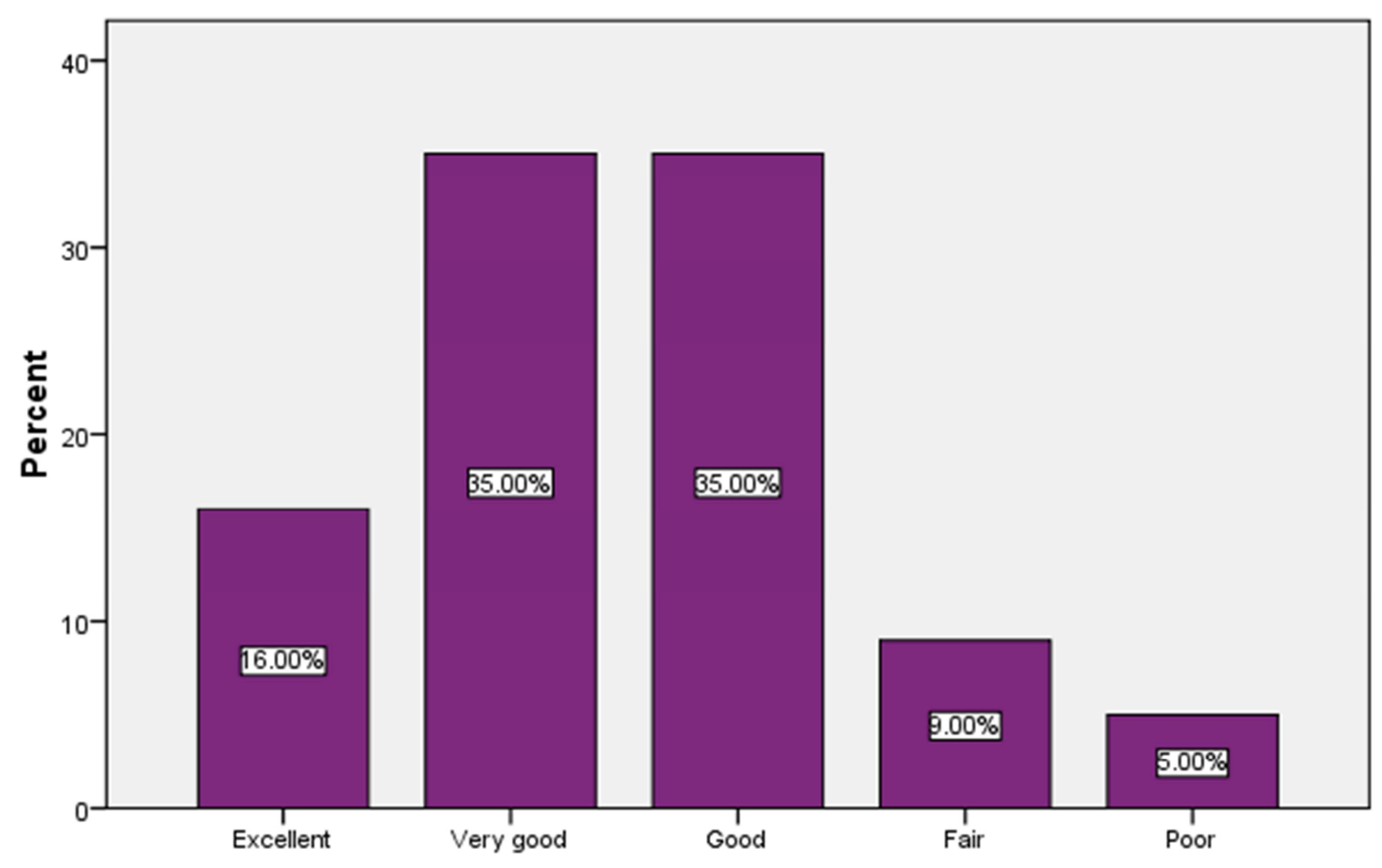

Rate of psychological support

Figure 1. Rate of psychological support. 


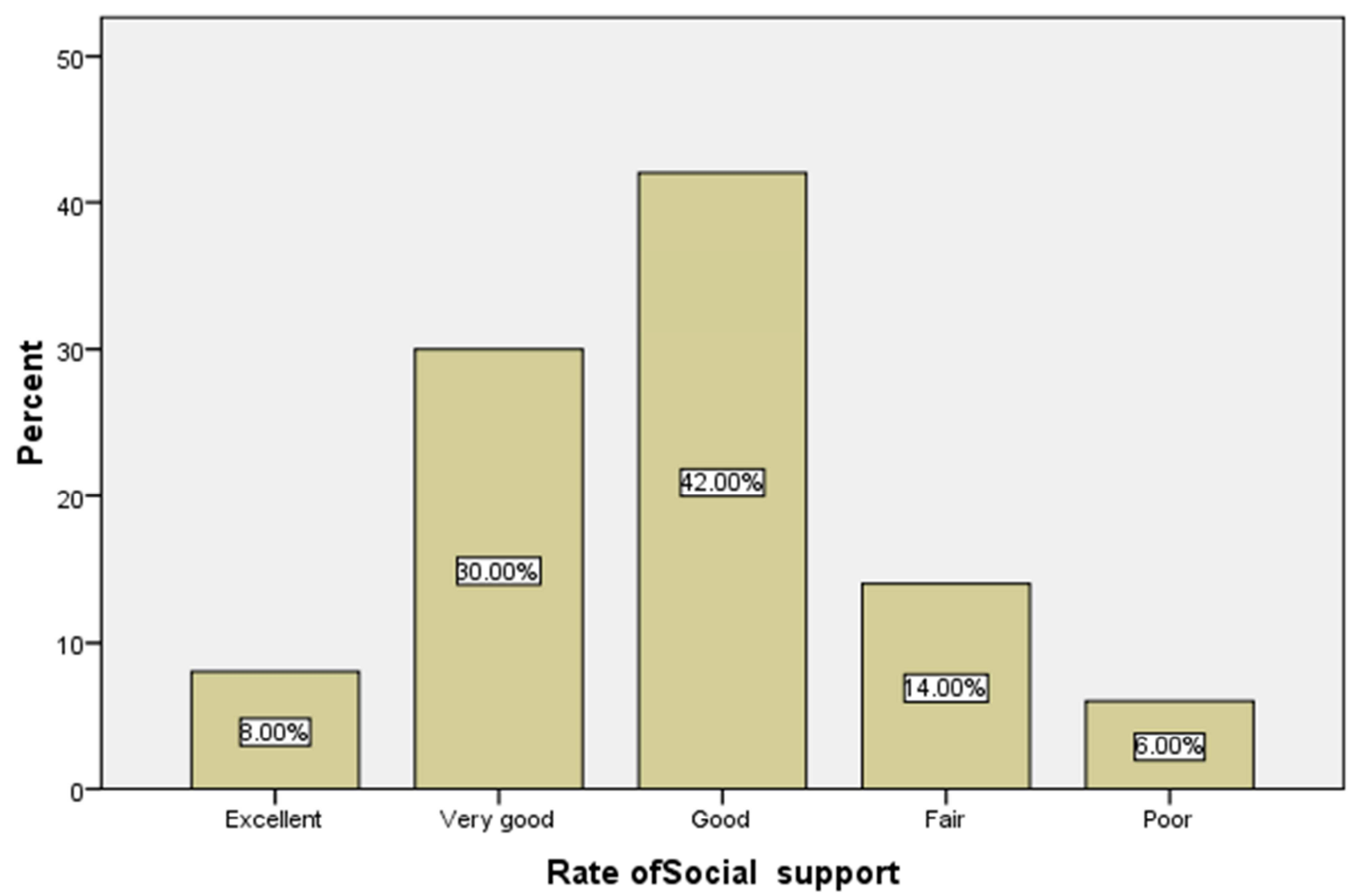

Figure 2. Rate of social support.

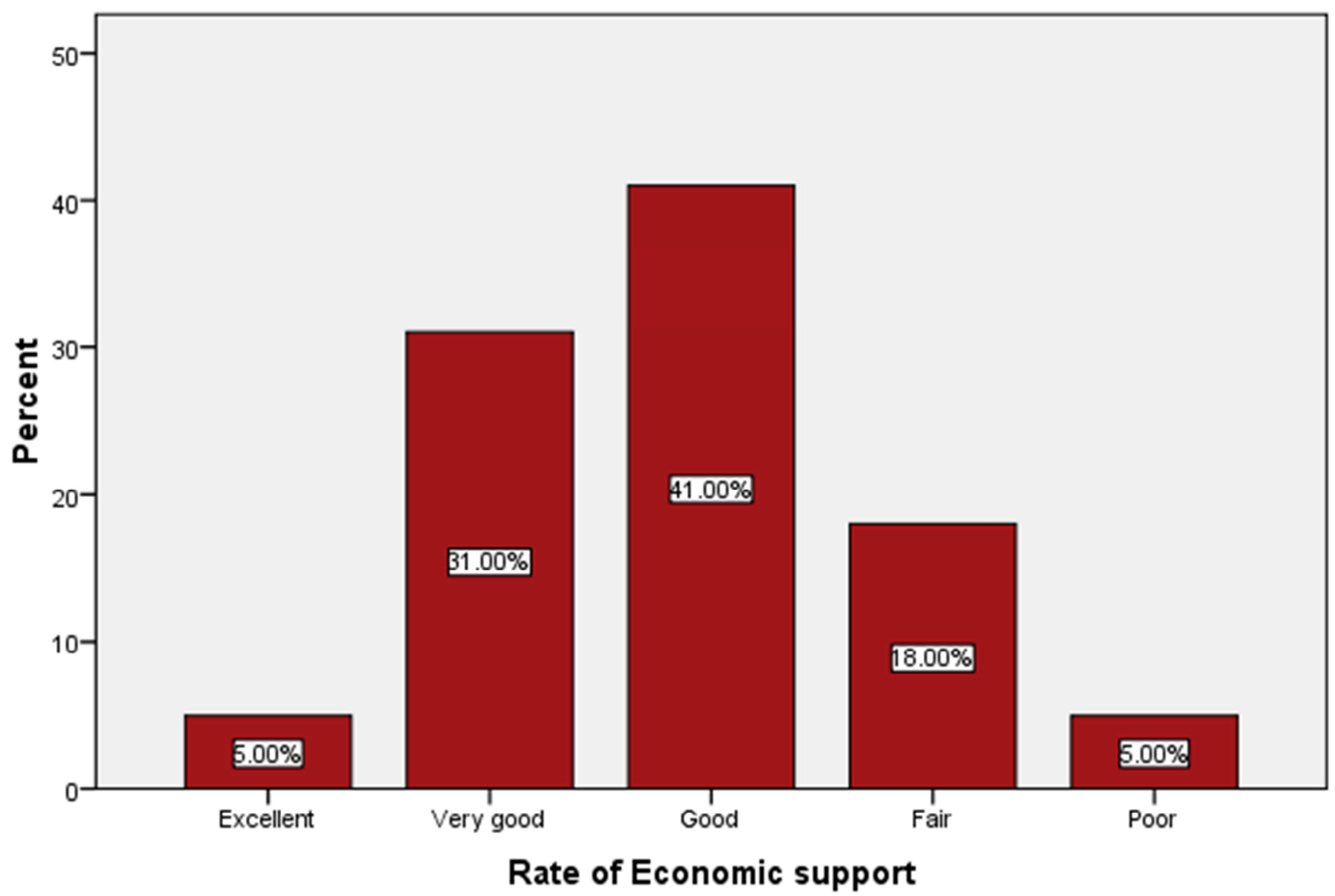

Figure 3. Rate of economic support. 


\subsection{Discussion}

The findings from this study indicate that the psychosocial, economic support services and demographic factors affecting the prognosis of schizophrenia are as prevalent as the research literatures indicate. The resulting data closely mirrored national statistics for what is intended to measure, which suggests that the group that responded reasonably matches the national population as it is corresponded in the literature. As was stated in literature, (3) the prevalence of schizophrenia in this study is nearly equal among male and female. Accordingly, the results from the study indicated that 51 male and 49 female respondents live with schizophrenia disorder. Similarly early age onset of schizophrenia is an indicator of poor prognostic in which this study reveals $30 \%$ of them are below the age of 25 years where psychosocial stability is not maintained. Schizophrenic clients with early onset of the disorder suffer from psychological immaturity, lack of social interaction and economic difficulties worsen the problem and affect the prognosis of the schizophrenic disorder. The schizophrenic client is, therefore, prone to be dependent on parents who have scarce knowledge about the disorder. When the head of a household who is the sole bread winner of the family develop schizophrenia the whole family drifts to extreme poverty and destitution.

Employment status is considered an important measure of disability in schizophrenia, and the extent to which societies offer opportunities for gainful employment, and thus, social reintegration is thought to be associated with course and outcome (14). In the literature of international study by world health organization it revealed that $70.3 \%$ of them are unemployed. In India 13\%, Multisite 20\%, Brazil 50\%, and Indonesia $41.3 \%$ and in Ethiopia $45.3 \%$ of schizophrenic clients are unemployed. In this study $52 \%$ of them are unemployed, and $1 \%$ dismissed. In Brazil unemployed clients will receive sickness benefit whereas this is not accustomed in our country. Unemployment and dismissal is among the psychosocial and economic supply service affecting the prognosis of schizophrenia. Managers and different authority person consider schizophrenic client as ineffective in their work and dismiss them. But this is a debate among managers even though schizophrenic clients are competent to accomplish many tasks assigned to them if they take their medication properly.

According to international study of schizophrenia, 2012, Persons with schizophrenia have been found to have low rates of marriage. As a consequence, there is often an implicit assumption that high rates of marriage among persons with schizophrenia are an indicator of sociocultural environments that promote better course and outcome. Rate of marriages are relatively high in Ethiopia $70.0 \%$, Nigeria $60.8 \%$, Indonesia $63.0 \%$, and Chennai 60.9 , and India $69.7 \%$. In this study, however, only $34 \%$ of schizophrenics are married. Furthermore, separation and divorce were fairly common for subjects in a number of cohorts, for example, Brazil 15.4\%, Ethiopia $17.9 \%$, and Chennai $39.6 \%$ and much higher than those of general populations. A retrospective study in Nigeria found that $27.5 \%$ of the sample had experienced marital breakup while $39.2 \%$ had never been married. In this study $49 \%$ of schizophrenics are single, $13 \%$ separation and divorce, and $3 \%$ of schizophrenics are widowed, remind psychosocial and economic support services and demographic factors affecting prognosis of schizophrenia. Singleness, separation and divorces are the psychosocial support services and demographic factors that determine the prognosis of schizophrenia for the spouse and children. Marriage lessens worsening of schizophrenia disorder in every perspective.

In Ethiopia clients with a diagnosis of schizophrenia who were nearly all participants $97.4 \%$ expressed agreement to at least one stigma item contained in the internalized stigmata at a time of interview; $46.7 \%$ had a moderate to high mean stigma score which is similar to a study made by internalized stigma among patients with schizophrenia in Ethiopia, [14]". Over $60 \%$ of the variation in the experience of stigma was explained by four factors: social withdrawal (16.7\%), perceived discrimination (14.1\%), alienation (13.9\%) and stereotype endorsement (12.7\%), [14]". In this study from the total $16 \%$ of schizophrenic client who experienced stigma, social withdrawal is $43.75 \%$, alienation is $25 \%$, perceived discrimination is $18.75 \%$ and stereotyped endorsement is $12.5 \%$. As a consequence $31.25 \%$ of them are socially withdrawn, $25 \%$ of them are alienated, and $6.25 \%$ of them perceived discrimination, and $37.5 \%$ of them suffer from stereotyped endorsement. As a result of stigma associated with schizophrenia clients evacuated from job, exposed to non-daily income, divorced and finally stop taking the prescribed medications. Once the prescribed medication are discontinued returning client to pre level of functioning is common and time consuming and exhaustive to recover again.

Discontinuation of medication is the consequences of psychosocial and economic support services problem that affect prognosis of schizophrenia. In this study $43 \%$ of the respondents are discontinued their medication at least once during their follow up period. Reasons for discontinuation of medications are $35 \%$ client's need of traditional therapy, $23 \%$ is due to lack of money, $7 \%$ of them discontinued prescribed medication due to unavailability of ordered medication, and the other $35 \%$ is due to other factors such as increased price of medication in the market, feeling hopelessness on the outcome of the disorder. In a cross-sectional facility-based study by [14]" it appears that almost half of those who discontinued their treatment reported that they had done so because of perceived stigma.

Daily and monthly income is a crucial importance for the survival of schizophrenic clients especially related to their illness. Accordingly $74 \%$ of schizophrenic client monthly income is below $\$ 27$ (below one dollar per day), 16\% of them are in between $\$ 27$ and $\$ 50$ and the remaining $10 \%$ of them earn above $\$ 50$ per months.

Concerning functioning of schizophrenic clients, the premorbid functioning of schizophrenic client reply $79 \%$ of them are good functioning and $21 \%$ of them are not 
functioning according to data gathered from 100 respondent schizophrenic clients. But in the current schizophrenic functioning $15 \%$ of them respond very good functioning, $45 \%$ good functioning and $40 \%$ of them are not functioning. This implies that schizophrenia impairs personal functioning and delay illness prognosis.

In the data collected from 100 schizophrenic clients at Adama Hospital medical college psychiatric clinic, the rate of psychological, social and economic support is greater than $50 \%$ very good and good and only $5-6 \%$ of the respondents rate the support as poor and very poor. According to the gathered data, people primarily offer psychological support and then followed by social support and lastly economic support.

The study generalize that lack of education, unemployment, unhappy marriage, less monthly income, stigma and discontinuation of prescribed medications are among the psychosocial, economic support services and demographic factors affecting the prognosis of schizophrenia disorder. Family, friends and significant others should not become overwhelmed with feelings of frustration or failure due to clients delayed prognosis. Although it is prevalent, prognosis of schizophrenia disorder is seldom a topic of conversation among parents and others due to the private nature of the topic and perhaps to spare the feelings of the client. In offering psychological, social and economic support the contribution of the whole members of the society is the only remedy. As stated earlier, this paper is an attempt to inform the reader about prognosis of schizophrenia disorder and lists psychosocial, economic support services and demographic factors affecting treatment methods and parent practices; it by no means should be a substitute for professional care.

\subsection{Conclusion}

As can be seen from the study findings, the psychosocial, economic support services and demographic factors affecting the prognosis of schizophrenia are early age of onset, low level of education, unemployment, dismissal, un happy marriage, lack of insight about the disturbance, insufficient family, friends and significant other contribution in counseling service, and Stigma with its consequences. Finally the economic factors that affect the prognosis of schizophrenia are minimal daily and monthly income, discontinuation of the prescribed medication due to lack of money, increased price of antipsychotic medication and insufficient financial support in any important part of client's life.

\section{Acknowledgements}

There are many people I wish to thank for taking part in helping me to achieve my academic goals. First and foremost my deepest gratitude is to my advisor Dr Kumsa Donis for his criticism and insightful comment from the inception to completion of this study. Without his comment and advice the completion of this paper is impossible. I would like to thanks Adama Hospital Medical College social and public health department, all staffs at psychiatric department and my parents, for your supports, for your patience, and for always encouraging me to do my best.

\section{References}

[1] Bootzin R, and Acocella J, [1996]. Abnormal psychology, Fifth edition. University of Arizona. USA.

[2] Kaplan HI and Sadock B, [2007]. Synopsis of psychiatry: Behavioral science (clinical psychiatry) $10^{\text {th }}$ edition.

[3] Kaplan HI and Sadok B, [1988]. Pocket hand book of clinical psychiatry: $2^{\text {nd }}$ edition New York.

[4] Alem A, Fikadu A, Kebede D, Shibre T [2004]. Bipolar disorder among an isolated island community in Ethiopia, Journal of Affective Disorders. Ethiopia.

[5] Alem A and Yitayew Y, [2007]. Psychiatry for primary health care practitioner in Ethiopia: Addis Ababa University. Ethiopia.

[6] Kebede, Alem A, Shibre T, [2004]. Schizophrenia Research: Addis Ababa University. Ethiopia.

[7] American Psychiatric Association, [2014]. Diagnostic and Statistical Manual of Mental Disorders: $5^{\text {th }}$ edition, Arlington.

[8] American psychiatry association, [2013]. Diagnostic and statistical manual of mental disorder: $4^{\text {th }}$ edition revised, Washington De 5.

[9] Thomson and Logo S, [2008]. Gale encyclopedia of mental health, second edition. China.

[10] Gorczynski P and Faulkner G, [2010]. Exercise therapy for schizophrenia, Cochrane Database of Systematic Reviews: PMID.

[11] Jablensky A, Sartorius N, Ernberg G, [1992]. Schizophrenia: manifestations, incidence and course in different cultures, A World Health Organization ten-country study, Psychological MedMonogram.

[12] Vanessa Taylor, [2011], Prevalence of schizophrenia: National institute of mental health. US.

[13] World Health Organization, [2001] mental health: new understanding, new hope, World Health Organization: Geneva.

[14] Assefa, D, Shibre, T [2012]. International stigma among patients with schizophrenia in Ethiopia: a cross sectional facility based study. BMC psychiatry. 